\title{
i
}

\section{Poesia, identità e trilinguismo in Amelia Rosselli Maria Pacella}

\begin{abstract}
In Amelia Rosselli la ricerca della "propria" lingua si è attuata con rigore e tensione drammatica, ma anche con gioco e leggerezza, coincidendo con la ricerca della sua stessa identità. Tra aperture e chiusure la lingua rosselliana si costruisce con solidità di corpo e svela la sua origine trilingue.

PAROLE CHIAVE: Amelia Rosselli (Parigi 1930 Roma 1996); poesia; identità; lingua, trilinguismo.
\end{abstract}

You self-taught heart who has understood its weak point, obduring through winters too great for harmony. You tent of oxygen you pale brown orange-leaved overhauling tough with your pen you may think, and analyse encounters or things into being, they escape you not without poverty, they take your hand and beg: express me!

O cuore autodidatta che ba capito il proprio punto debole, che resisti caparbio a inverni troppo rigidi per l'armonia. $O$ tenda a ossigeno o bruno pallida revisione a foglie arancio quantunque tu possa pensare con la tua penna, e analizzare incontri e cose fino a farle esistere, esse ti sfuggono non senza povertà, ti prendono per mano e ti supplicano: esprimimi! 
Poesia, identità, trilinguismo sono temi forti nella vita e nell'opera di questo poeta $^{1}$ che, nata a Parigi nel 1930 da padre italiano e madre inglese, subi a sette anni il trauma della perdita precoce e violenta del padre Carlo Rosselli, militante antifascista, ucciso insieme al fratello Nello da sicari francesi, su mandato di alte gerarchie fasciste italiane.

Questa perdita iniziale, vera e propria tragedia familiare, segnò lo svolgersi della sua vita. Nel 1940, a dieci anni, Amelia si trasferì con la madre in Inghilterra prima, e negli Stati Uniti poi, per tornare in Italia nel '46; nel '48 di nuovo tornò in Inghilterra per studi di musica e composizione; a 18 anni, morta la madre - un'altra perdita precoce e durissima - torna in Italia, lasciando i fratelli in Inghilterra, a Firenze, dalla nonna prima e poi, negli anni '50, va a Roma dove visse fino alla sua morte, avvenuta anch'essa in modo tragico e violento, come quella del padre, non per omicidio, ma per suicidio: Amelia si uccise lanciandosi nel vuoto dalla finestra

1. Scoperta da Pasolini nel ' 63 , Amelia Rosselli ha pubblicato nel ' 64 e nel '76 le due maggiori e più importanti raccolte intitolate, la prima Variazioni belliche e la seconda Documento.

Oltre che Pasolini, di lei si sono occupati critici-poeti come Giudici, Zanzotto, Raboni e critici-poeti donne, soprattutto di Roma, la città in cui visse per gli ultimi quarant'anni della sua vita, a lei legati da rapporti di amicizia o di frequentazione, come Antonella Anedda, Daniela Attanasio, Maria Clelia Cardona e molti altri critici letterari donne che hanno grandemente contribuito ad illuminare la sua poesia e la sua vita. Altri critici di cui ha richiamato l'attenzione sono stati Alfonso Berardinelli, Pier Vincenzo Mengaldo, Giulio Ferroni, solo per citarne alcuni, oltre che critici stranieri. Tuttavia, nonostante i giudizi positivi, ed alcuni entusiastici e ammirativi, la sua opera non ha ricevuto ancora una lettura attenta e approfondita perché la difficoltà $e$ l'inclassificabilità della sua scrittura mettono in scacco molti critici. Su questa questione si veda come si esprime Giulio Ferroni nell'articolo "Un' 'altra' vita perduta", in Galleria, n.1/2, 1997, p. 24: "Ma di fronte a questa poesia anche il rentativo della critica di razionalizzare, di definire, di motivare, rischia di sfuggire a se stesso: il grande rilievo dell'opera di Amelia Rosselli è mostrato anche dal modo in cui, senza pretese programmatiche, viene a sfidare la spinta alla semplificazione che inevitabilmente si insinua nell'atto del critico-lettore. Il critico deve imparare ad inseguire le sue folgorazioni assolute, di cui può avvertire la forza e l'evidenza, la lacerante alterità: ma non può pretendere di ridurle a formule, a schemi, a modelli onnicomprensivi. Esse gli impongono di riconoscere la propria insufficienza, forse gli chiedono di rivedere i propri schemi mentali, la credibilità dei propri presupposti teorici e delle proprie ossessioni metodologiche, il proprio stesso modo di inseguire il manifestarsi della poesia e del linguaggio in questo mondo". 
del piccolo appartamento in Roma, in cui viveva da sola, l'11 febbraio del 1996. Aveva 66 anni.

Dunque: Francia (da 0 a 10 anni), Inghilterra e Stati Uniti (da 10 a 18 anni), Italia (da 19 a 66 anni). Tre lingue tutte insieme fin dall'inizio: il francese - la lingua della nazione in cui è nata, l'inglese - la lingua della madre, l'italiano - la lingua del padre. Praticamente tre lingue madri e, dietro, tre culture, tre/quattro nazioni, tre letterature. E anche molte religioni: ebrea per parte di padre, quacquera, cristiana e buddista per parte di madre, lei stessa, semmai, buddista. Molte radici, molti mondi ${ }^{2}$ e, soprattutto, molte lingue che si parlano dentro di lei e che certamente hanno contribuito a complicare - ed arricchire - il già difficile rapporto che "questa specie di apolide" (come l'ha definita Pasolini) intratteneva con se stessa ${ }^{3}$.

Amelia, infatti, fin da bambina, forse per il trauma della visione che ebbe a 7 anni del corpo del padre sfigurato a coltellate, iniziò a soffrire di manie di persecuzione che, a volte, diventavano dei veri e propri deliri ossessivi che l'avrebbero tormentata per tutta la vita e che le avrebbero dato un'instabilità e un livello di sofferenza tali da consumarla e da renderle insopportabile la solitudine da cui il fare poesia contribuiva a tirarla fuori e a metterla in contatto con il mondo 4 .

Dunque un'instabilità psichica resa forse più precaria dalla triplice identità culturale e linguistica. La sua identità una e trina, proprio come la S.S.Trinità della

2. Cosl si descrive Amelia in una sua poesia: "Nata a Parigi travagliata nell'epopea della nostra generazione / fallace. Giaciuta in America fra i ricchi campi dei possidenti / e dello Stato statale. Vissuta in Italia, paese barbaro. / Scappata dall'Inghilterra paese di sofisticati. Speranzosa / nell'Ovest ove niente per ora cresce." Amelia Rosselli, Variazioni belliche, in Le poesie, Garzanti, 1997, p. 202.

3. Forse hanno anche contribuito ad aumentare il suo senso di estraneità e differenza rispetto agli altri a causa del disagio psichico.

4. A questo proposito è interessante notare che non ci sono poesie dedicate alla morte del padre (il trauma, rimosso, non può essere detto), ma questa morte è dietro tutte le poesie e dietro tutta la sua vita. Si veda questa folgorante notazione sulla scrittura tosselliana fatta dal poeta e critico Antonella Anedda: "l'assenza arde $i$ bordi delle parole lasciando una voragine di cenere". In Antonella Anedda, "Amelia Rosselli. L'attraversamento della notte". Articolo-saggio in occasione del Premio Maria Attanasio. 
religione cattolica, è il tema di questa poesia in inglese, in cui, nel finale, il misticismo di Amelia si capovolge nel suo rovescio comico:

O were I one in Three! Just like the Holy Ghost, the Father and the Son, I'd reunite my scattered souls and string them in from all the seas abroad;

no longer climb upon perdition's mast

and wave a banner crying God, at last!

Se è vero che "riconquistare la propria (o, comunque, una propria) lingua come una lingua straniera, liberata dall'usura dell'abitudine e pertanto investita di una più intensa potenzialità comunicativa, è privilegio e anche arduo compito del poeta", per Amelia questo è stato vero in senso letterale. A lei, in qualche modo, le tre lingue dovevano apparire familiari e straniere nello stesso tempo: "lingua corrente? in all 3 langues (anyuse?)"

La ricerca di una propria lingua poetica in lei si è intrecciata, e in parte è coincisa, con la scelta di una lingua fra tre lingue-madri e il fatto di non potersi identificare naturalmente con una ha aumentato, forse, il suo senso di consapevolezza della lingua come strumento espressivo e, nello stesso tempo, il suo distanziamento da essa - la lingua come Altro da sé - che ha eccitato e stimolato le sue capacirà creative.

A questo punto si pone un problema critico importante e di non facile soluzione, di cui nel presente lavoro si vogliono indicare alcune possibili linee di sviluppo: che peso abbia avuto il trilinguismo nella lingua poetica della Rosselli. Per una scrittura

5. "O foss'io una in Tre! Proprio come lo Spirito Santo / il Padre e il Figlio, radunerei le mie sparse anime / e le legherei insieme da tutti i mari dell'estero / non più arrampicarsi sull'albero della perdizione / ma sventolare una bandiera gridando Dio, infine!". (trad. di A. Anedda). October Elizabethans, in Amelia Rosselli, Primi Scritti 1952-1963. Guanda, 1980, p. 57.

6. Giovanni Giudici, prefaz. a Amelia Rosselli, Le poesie, op. cit., p. 10.

7. Amelia Rosselli, Diario in tre lingue in Primi Scritti 1952-1963, op. cit., p. 104. 
così creativa come quella di Amelia, in cui la trasgressione diventa la regola, individuare quali tracce vi abbia lasciato il trilinguismo non è impresa facile, iniziando da alcuni problemi: individuare, ad esempio, quali lapsus, quali "errori", siano voluti e quali no, definire la presenza del francese e dell'inglese nell'italiano e, in generale, il rapporto fra le tre lingue, chiarire la natura dei vari scarti linguistici, degli anacoluti, delle ambiguità paronomastiche, così caratteristici di questa poesia.

$\mathrm{Ma}$ forse può risultare fuorviante o riduttivo mettersi su questa strada, trattare questo problema critico solo, o principalmente, come un problema linguistico, se è vero che la straordinaria lingua poetica di Amelia è una lingua a metà strada fra conscio e inconscio, che è "uno spazio di rivolta contro le consuetudini mentali e le convenzioni linguistiche", che nella sua opera "c'è soprattutto una forma mentale, un'attitudine a destrutturare e insieme a ricomporre nel desiderio un universo linguistico", che "il suo lavoro linguistico è veramente una questione di vita o di morte"10. Anche se è vero che una lettura attenta delle singole opere potrebbe rivelare delle "regole" di questo lavoro di destrutturazione che aiuterebbero a penetrare nella confusione così significante di questa poesia.

La sensibilità linguistica di Amelia, la sua percezione della lingua sono molto particolari: le parole le apparivano da fuori, come un involucro, nei loro aspetti più fisici e sensuali, come oggetti dotati di colore, sapore, suono. ${ }^{11}$

8. Daniela Attanasio, "Ad apertura", in Galleria, op. cit, p. 7.

9. Antonella Anedda, Amelia Rosselli. Lattraversamento della notte, op. cit..

10. Stefano Agosti, apud Emmanuela Tandello, "Introduzione", in Galleria, op. cit., p. 9.

11. Per notare come questa lettura delle parole fosse in lei prevalente, si veda quanto Amelia scrive nelle "Note" a "La libellulaw: "Il tirolo La libellula vorrebbe evocare il movimento quasi rotatorio delle ali della libellula, e questo in riferimento al tono piutrosto volatile del poema La libellula pud anche ricordare le parole alibellon, "libertàn: infatti il mio poema ha come tema centrale la libertà, e il nostro, e mio, ulibellarla..." E interessante notare l' associazione che il poeta fa tra il movimento delle ali della libellula e il "tono... volarile del poema" (l'autore non legge la libellula come simbolo della poesia, della libertà della poesia, interpretazione a cui abilita lo stesso testo $\mathrm{e}$ che sarebbe la lettura più normale) e lo scivolamento di significati che il poeta fa a partire dal 


\section{Chi l'ha conosciuta e frequentata abitualmente, le sue amiche, poetesse romane,} che l'avevano presa in cura e tentavano di alleviarle la solitudine e la sofferenza in cui viveva rinchiusa, parla della sua anima bambina ${ }^{12}$, della capacità e del piacere di deformare le parole dell'uso quotidiano riflettendovi sopra con un processo di estraniamento e masticandole fino a crearne delle nuove, quasi usando le parole come giocattoli. Questo gioco dell'invenzione linguistica, il bisogno di forzare i limiti oggettivi di una lingua per crearne una sua propria e personalissima hanno trovato un terreno fertile nella sua radice trilingue.

Io credo che anche - o soprattutto - da qui bisogna partire, dalla natura più profonda dell'autrice, dal suo temperamento, dal suo modo di essere ${ }^{13}$ per spiegare

rermine libellula: libello, libertà, libellare[la] (inventato), del tutto indebito da] punto di vista etimologico. Il fatto che lei adotti questa lettura delle parole, basata sull'evocazione, sui suoni, quasi un gioco di filastrocca, in un testo critico, fa capire come questa visione e quest'uso della lingua fossero normali per lei, come si evince anche da molte sue poesie.

12. Questo lato del carattere di Amelia, adombraro in articoli scritri su di lei dalle sue amiche poetesse, è stato poco analizzato dalla critica, ma forse riveste un importanza fondamentale per capire molti aspetti della sua poesia: la passionalità irruente, la vivace curiosità, la rabbia e il furore, la tensione erotico-religiosa, il misticismo e la fede naturale, la violenza e la tenerezza, la leggerezza e la gravità, l'invenzione linguistica e le iterazioni come filastrocche o cantilene. Qualche accenno si può trovare in Alfonso Berardinelli, "Prefazione" a Diario ottuso, Empiria, 1997, p. 7: “Seria, grave e totalmente impegnativa come un gioco di bambini, (corsivo mio)... la scrittura di Amelia Rosselli non ha la felice instabilità del flusso, è allarmante e intermittente." E più avanti: "E ogni "perché» rivolto alla vira è un pozzo senza fondo... Tutto lo spazio della vita di tutti $i$ giorni sembra perforato dal terribile rintocco dei "perchén: come se non ci si poresse rassegnare o pacificare nella certezza, nella stabile convinzione convenzionale di essere qui." (pp. 8-9). Non sono cos̀̀ i "perché» dei bambini? Dietro le loro domande impossibili non si nasconde una richiesta di rassicurazione esistenziale?

13. Così ne parla il poeta-scritrore Maria Clelia Cardona nel suo articolo "Io non so se io rimo per incanto o per travagliata pena", in Galleria, op. cit., pp. 129-135: "Ho conosciuto Amelia verso la fine degli anni Sertanta. Era alta e magrissima, con una testa da ragazzo. Portava i capelli quasi rasari, come nessuna donna di cinquant'anni oserebbe mai. Aveva occhi celesti grandi e severi. Si pensava, vedendola, a una santa guerriera, a una Jeanne d'Are metropolitana. Quando si andava da lei la si trovava semisdraiata sul letto. Fumava, stringendo la sigaretta fra il pollice e l'indice e scuoteva via la cenere con il medio. Aveva un suo modo sollecito e distratto di accogliere le nuove conoscenze: storpiava i nomi, sembrava dimenticare tutto ciò che le veniva detro, ma 
il senso e il valore di una poesia così unica nella sua voce, così urgente e necessaria, in cui la grammatica e la sintassi vengono sistematicamente distorte, in cui la convenzione degli stili, dei generi, della metrica viene rifiutata e trasgredita, non tanto per influssi culturali ed artistici o per adesione a poetiche dell'avanguardia - da cui Amelia si tenne sempre distante - ma per ineludibile fedeltà a se stessa.

La definizione della sua scrittura poetica come "lapsus", data da Pasolini nel 1963 sul n. 6 del "Menabò" presentando la poesia di Amelia al grande pubblico - definizione con cui la poetessa aveva un rapporto conflittuale ritenendola forse più descrittiva che esplicativa -, ha iniziato una serie di interventi che sono stati spesso fortemente condizionati da questa definizione iniziale e molti critici si sono mossi

di ciascuno tratteneva nella mente almeno un granello intorno a cui costruire nel tempo amicizie o motivate esclusioni. [..] A quel tempo - erano i primi anni Ottanta - viveva con una pensione di trecentomila lire al mese. Era poverissima, cioc̀. [...] Fino a una quindicina di anni fa frequentarla era una prova difficile per chiunque: era rigorosa, aspra, intransigente, incapace di mediazioni, e andava a colpire con furore, come un angelo giustiziere, ogni atteggiamento compromissorio. Viveva in una dimensione assoluta, violenta, ironica e tragica. Strapazzava senza pietà chi presumeva di accostarsi alla poesia tenendo i piedi al caldo, per così dire. [...] Telefonava spesso per proporre qualche film. [...] Le piacevano i film di Kieslowski, di Rohmer, di Jane Campion; odiava i film pretenziosi e intellettualistici, come odiava la falsa letteratura dei ragionieri che non si sognerebbero mai di parlare e di vivere come scrivono. [...] Era spesso sola. La invitavano a fare letture di poesie, le facevano interviste, la inserivano nelle antologie, le assegnavano premi letterari. Tutto questo lei lo chiamava lavoro e vi metteva il massimo impegno: chi la ha ascoltata leggere le sue poesie sa bene quali altissimi risultati musicali - timbrici e ritmici - riuscisse a raggiungere con la sua voce fonda e roca. [...] A differenza di tanti altri, non amava parlar male del mondo letterario: il successo altrui non la ingelosiva, le trame di potere non la interessavano, l'insensibilità degli editori non la scandalizzava. Compariva mamente in TV o sui giornali: non c'era da meravigliarsene, perché troppo radicale era l'incompatibilità fra il linguaggio della sua poesia (che era il suo unico linguaggio) e quello televisivo e giornalistico. Ricordava spesso che agli inizi quattro editori avevano respinto i suoi datriloseritri. [..] Le piaceva raccontare come lavorava a quel tempo: «Suonavo Chopin e subito dopo scrivevox e mimava il gesto del suonare il piano e poi girarsi di scatto per muovere le dita sulla tastiera della macchina da scrivere come su uno strumento musicale gemello. Cosi faceva anche con Béla Bartòk e Webern. Diceva di sentire il verso come un elastico, da tendere fin dove poteva essere teso - $e$ anche qui faceva con le dita il gesto dell'allungarsi e contrarsi di una sottile striscia cedevole $e$ resistente.[...]" 
aggirandosi nel solco di questa tradizione, producendo interpretazioni riduttive e parziali ${ }^{14}$, che non intaccano la superficie di questa poesia.

La difficoltà della poesia di Amelia è anche la difficoltà di entrare in un'opera che si presenta come un corpo unico e compatto - o unica e compatra come un corpo? -, una presenza solida e impenetrabile. La sua poesia atterrisce e affascina con il suo magma insostituibile, avvicina ed allontana nello stesso tempo, sicuramente scuote e non lascia indifferenti. Ė una sfida al senso, una scrittura che si impone per la sua presenza, perché c'è, perché esiste, anche "senza comunicare" Il ritro, il tono, sono più importanti del significato ${ }^{15}$.

A ciò si aggiunga un altro elemento importante: la poesia di Amelia, pur così assoluta e incatalogabile, - o forse proprio per questo? - è una poesia profondamente sessuata. I continui riferimenti al corpo suo o altrui, i molti richiami all'uomo amato, il bisogno struggente di tenerezza e amore, l'acutezza e la sofferenza del suo sentire, parlano di un io lirico donna, squisitamente donna, che esibisce senza pudore il proprio vissuto ${ }^{16}$, facendone la radice stessa della propria poesia. In questo senso è uno degli esempi più potenti ed efficaci di una grandissima scrittura al femminile nel panorama del Novecento italiano ed europeo. Nella sua poesia si vede sempre la donna che l'ha generata. Non è un caso, forse, che, con un'unica eccezione ${ }^{17}$, i suoi lettori migliori e più acuti, quelli che meglio l'hanno compresa

14. Si veda, ad esempio, la presentazione della sua poesia fatta da Pier Vincenzo Mengaldo: - ... una scrittura, o piuttosto una scrittura-parlato, intensamente informale, in cui per la prima volta si realizza quella spinta alla riduzione assoluta della lingua della poesia a lingua del privato, che si ritrova quindi in non pochi poeti postsessantotteschi" che, francamente, mi pare limitativa e inadatta a dare ragione di una poesia come quella di Amelia Rosselli, in Poeti italiani del Novecento, a cura di Pier Vincenzo Mengaldo, Milano, Arnoldo Mondadori, 1978, p. 995.

15. Così Giovanni Giudici descrive la poesia di Amelia: Aun sistema dove il cosa-vuol-dire appare assai meno importante del dire in sé", nella "Prefazione" a Amelia Rosselli, Le poesie, op. cit., p. X.

16. Ce lo dice la stessa Amelia: "In verità tra la mia biografia e la mia poesia ci sono strettissimi rapporti", in EmmanuelaTandello, "Introduzione", in Galleria, op. cit., p. 10.

17. Alfonso Berardinelli, op. cit. 
come donna e come poeta, i critici più profondi, siano state donne - poeti, quelle stesse che in vita hanno cercato di circondarla dell'amore di cui era costantemente in cerca, che l'hanno conosciuta e frequentata e che più di altri sono in grado di decifrare il continuum di vita e poesia.

Si capisce bene, dunque, come in una personalità così complessa e problematica come questa che si è andati delineando, il trilinguismo, inteso come aspetto trilingue della sua formazione e della sua produzione, non possa essere isolato come un tema a parte e nemmeno possa essere assunto come l'elemento caratterizzante della sua opera, pena il fraintendimento e la banalizzazione. Si possono, però, fare delle osservazioni e avanzare delle ipotesi: gli scritti in inglese e francese occupano una parte limitata della produzione rosselliana, molto inferiore, quantitativamente e qualitativamente, alla parte italiana, e coincidono - con l'unica eccezione delle poesie in inglese di Sleep - con gli esordi e con la sua giovinezza. Da un certo punto in avanti, in parte contemporaneamente, e poi sempre più dagli anni ' 60 in poi, P'italiano viene scelto come la lingua della sua scrittura. Tuttavia le lingue rifiutate non scompaiono, ma emergono qua e là nelle sue raccolte maggiori bucando l'italiano in un tessuto linguistico già segnato da "errori" lessicali (i famosi "lapsus") e sintattici, e da paronomasie e sinonimie in funzione ambigua.

A differenza di altri poeti, che sono monolinguistici, Amelia, quasi con un senso di fedeltà a se stessa e al proprio vissuto multiculturale, come un marchio della sua identità, mantiene queste presenze linguistiche diverse dentro l'italiano, la lingua della nazione in cui scelse di vivere, ma anche la lingua del padre assassinato, scelta forse anche per dar voce a chi non poteva più parlare, per un senso di affettuosa giustizia e risarcimento. Come testimoniare per un morto.

In questo senso il trilinguismo va ad aiutare la costruzione di quell'edificio unico e spaventoso che è la lingua rosselliana - e in cui è consistita la creazione della sua poesia -, vera e propria sovralingua e metalingua.

Vediamo di delinearne alcune fasi.

Prima di approdare all'italiano come lingua definitiva della sua poesia - e all'Italia e a Roma come luogo in cui vivere - per circa un decennio Amelia scrive brevi 
testi nelle tre lingue, raccolti e pubblicati in seguito, nel 1980, sotto il titolo Primi Scritti 1952-63, da lei poi definiti "esercizi poetici prima di scegliere una lingua"18. L'oscillazione fra inglese, francese e italiano (da un testo all'altro e all'interno dello stesso testo) s'interromperà con la scelta finale della lingua italiana in Variazioni belliche del 1958-59/'61. Con un'unica eccezione: la raccolta Sleep, poesie in lingua inglese scritte fra il '53 e il ' 66 , anche se l'uso di questa lingua va considerato come una tappa nel percorso che ha nell'italiano la lingua d'arrivo.

Questi Primi Scritti sono prose poetiche e poesie scritte tra il 1952 e il 1963. Il primo testo, del ' 52 - l'autrice aveva 22 anni - è in inglese (lingua della madre); poi c'è un testo del ' 53 in italiano (lingua del padre); segue un testo del ' 54 in francese (lingua del paese in cui è nata); poi una raccolta di poesie francesi scritte fra il 1954 e il 1961; del 1954 è una raccolta di prose in italiano; del '55 una raccolta di prose in francese e italiano; del '56 sono poesie in inglese; del '55-'56 una raccolta trilingue in prosa-poesia; del ' 62 una raccolta di prose in inglese; infine, del ' 63 una raccolta di poesie in italiano. C'è dunque un girovagare fra le tre lingue prendendo come punto di partenza l'inglese, la lingua della madre, passando per l'italiano e il francese, per poi approdare all'italiano come lingua definitiva. ${ }^{19}$ Si tratta di brevi opere di carattere fortemente sperimentale in cui l'autrice forza i limiti di ogni lingua, quasi a saggiarne le capacità espressive.

Qui ci si soffermerà su una delle più significative, anche perché più lunga, opera dichiaratamente, programmaticamente trilingue fin dal titolo, Diario in Tre Lingue, del 1955-56.

$E$ un diario sui generis, del tutto inclassificabile, in poesia-prosa in cui si mescolano le lingue, le letterature, $i$ generi, gli autori, $i$ ricordi in un gioco straniato $e$

18. Corsivo mio.

19. E interessante notare che questa ricerca avviene, in parte, contemporaneamente alla composizione delle poesie di Variazioni belliche, scritte fra il ' $58 \mathrm{e}$ il ' 61 . Questo significa che, mentre da un lato Amelia scrive la sua prima grande opera in italiano, dall'altro, continua, in parallelo, a scrivere nelle altre due lingue e che l'affermazione dell'italiano e la rinuncia alle altre due non sono state facili né tantomeno scontate. 
straniante senza né capo né coda. C'è di tutto in questo diario: il gioco linguistico come divertissement e nonsense in cui Amelia si diverte a distorcere i suoni e i significati passando da una lingua all'altra:

la goutte qui coute (-écoute)...

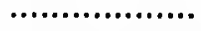

la lune sale qui sale $e^{20}$

au ciel plomb fingateau

fingatò

fingatos

fingatoeu

fingatoau

les arbres ciel pengateau

ciel pan gateau

ciel pengato

pengâtò

pengatoe

pengateo ${ }^{21}$

com plete nonsense

con pleat non sense

con pleat $9^{\circ}$ cence $^{22}$

le vin bralé

le vin buscule $e^{23}$

20. Valga una sola traduzione come esempio: "la goccia che costa (-ascolta)... la luna sporca che sale" (traduzione mia). Amelia Rosselli, Diario in tre lingue, in Primi Scritti 1952-1963, op cit., p. 71.

21. Ib., p. 78.

22. Ib. p. 80.

23. Ib., p. 87. 
C'è critica letteraria in versi, riflessioni personalissime sugli autori che la giovane Amelia andava scoprendo e amando, come il suo Montale, appunti di studio in cui la disposizione grafica ha la sua importanza. L'attenzione è rivolta ai suoni, agli accenti, alla metrica con intuizioni di rara sensibilità:

Montale-

procedimento surrealista non nelle immagini (le

quali sono realiste, minute) ma nell' accostamento delle

vocali, non abusato, controllatissimo ${ }^{24}$

Avrei voluto sentirmi scabro ed essenziale.

ecc.

Lo sai debbo riperderti e non posso

A.

ritmo Montale -

La religiosità intensa (trad. da Inglese; Bibbia)

(endecasillabo forma religiosa?)

di solito

a 3 accenti ( 2 se fuori testo)

come un tiro aggiustato mi sommuove ${ }^{25}$

Altrove la riflessione sui valori ritmico-metrici del verso conduce a risultati originalissimi e illuminanti: la poesia diventa un volume/cubo spazio-temporale dalle mistiche risonanze ${ }^{26}$ :

24. Ib., pp. 72-73.

25. Ib., p. 101. Le sottolineature sono dell'autore.

26. In questo pensiero teorico-mistico che si nutre di visioni, e poeticamente organizzato, mi sembra di trovare una certa analogia con quello di Walter Benjamin, un pensatore con cui Amelia condivideva l'origine ebraica. 
gli accenti sono le travi costruttorie del

cubo: - lo provocano. piazzamento accenti

provoca variazioni dinamiche (volume, ritmo,

piazzamento verso) nell'interno del cubo.

veux- 뜨

aller chez le prince?

il tempo nella poesia diventa volume del cubo; ciò̀

profondità tramite le attesa-spazio tra verso e verso. ${ }^{27}$

I nomi di molti altri artisti passano in questo Diario: Jung, Eliot, Proust, Penna, Campana, Rimbaud, Gauguin, sempre usati in modo del tutto originale, inseriti in un contesto poetico-sperimentale plurilinguistico. Valga come esempio:

Montale-Proust

italian stornello popolare

greek-latin prose

Joyce, frantumazione

Surrealismo (french)

classici

argots

Chinois

strutture lingue straniere

eliot-religious ${ }^{28}$

in cui, in una sorta di cocktail trilingue, vengono accostati autori delle tre letterature, mondo greco-latino, cinese, argot (la lingua di strada francese), tutto insieme in un calembour, in cui lo stridere delle differenze e l'accordo delle analogie sono parte del gioco producendo effetti di senso.

27. I6., p. 101.

28. Ib., p. 76. 
Ma, dietro il multiculturalismo linguistico e letterario, parte di esso, compaiono i morti: la madre Marion Cave è qui ribattezzata "Marion-l'eau qui coule", "Marion-l'acqua che scorre", forse una "Marion-pianto continuo", divenuta tale dopo la perdita prematura e atroce del marito, e, subito dopo, "Marionlot", "Marionstatuadisale" con evidente riferimento al Lot della Bibbia trasformato in statua di sale dallo sguardo della moglie; qui l'analogia è per contrasto: è Marion, la moglie di Carlo Rosselli, pietrificata dal suo stesso sguardo sopra il corpo straziato del marito.

E, solo due pagine dopo, in modo analogo, "Zio Nello (petite notion autobiographique nécessaire)"29 si trasforma in "sionel" dal Monte Sion, collina di Gerusalemme, simbolo del mondo ebraico, ancora un riferimento alla cultura ebraica come richiamo alla propria storia familiare.

In ambedue i casi lo sperimentalismo, il gioco deformante dei nomi distanziano le persone oggettivandole in personaggi, raffreddando il ricordo della tragedia e la sofferenza della perdita.

Dietro questi usi linguistici si intravedono le suggestioni letterarie: le avanguardie novecentesche, i surrealisti, Joyce, ma, al di là di queste, questo passeggiare in libertà nelle tre lingue, distorcendo fondendo variando, mette in risalto il livello affetrivo delle lingue. Il gioco e l'invenzione fanno risaltare l'intimità che Amelia aveva con tutte e tre le lingue facenti parte della sua identità, della sua esperienza di vita.

A volte esse sembrano confondersi e intrecciarsi fra di loro nello stesso rigoverso, o addirittura nella stessa parola, come avviene nella parlata degli emigranti, quale lei era.

Insomma, nel momento in cui Amelia scrive il suo "diario", questo non può che essere trilingue! Nello stesso tempo appare chiaro, però, come per lei il trilinguismo sia stato uno stadio da superare, un passaggio obbligato per arrivare al monolinguismo delle sue maggiori raccolre, anche se le due lingue rifiutate, l'inglese e il francese,

29. "piccola notazione autobiografica necessaria" (traduzione mia). Da notare lo scivolamento ironico che distanzia dall'oggetro. 
fanno capolino all'improvviso nei versi e forse, indirettamente o inconsciamente, contribuiscono alla creazione dei "lapsus" in italiano ${ }^{30}$.

Non sono molte queste presenze straniere, la maggioranza in francese, e tuttavia marchiano la lingua poetica della Rosselli. Vediamone alcuni esempi:

E poi si adatterà, alle mie cambiate contingenze, car io ho cambiato residenza, non sono più il fiore timido appeso dove erano i salici e non voglio le tue tenerezze...

Contro del magazziniere si levava il grido dell' incoscienza contro del pourboire coniavo un'altra frase, quella dell'incertezza. ${ }^{32}$

Amare frasi andai ripetendo finché non ti rintracciai

Sul tuo trono di viande e di disperazioni. ${ }^{33}$

Premi il tuo disingaggio nella notte rivedi i programmi, amour je tai tute notte di nuovo le caramelle una lavagna io ti scorto nelle tue dita misogene $e^{34}$.

tuo motivo non urlare, dinnanzi alla cattedrale; esilio o chance non ti perdonano la locomotrice ${ }^{35}$.

Sminuito impero, non era sicuro di poter tentare il tragitto

30. Su questo tema Emmanuela Tandello parla dell' importanza fondamentale che la formazione letteraria cosmopolita e l'apprendistato trilingue ricoprono per lo sviluppo del linguaggio poetico rosselliano", nella nota introduttiva ad Amelia Rosselli, Le poesie, op. cit., p. XV.

31. Amelia Rosselli, Variazioni belliche, in Le poesie, op. cit., p. 165, "car" trad. "perche"

32. 16., p. 207, "pourboire" trad. "mancia".

33. I6., p. 320, "viande" trad. "carne".

34. Amelia Rosselli, Serie ospedaliena, in Le poesie, op. cit., p. 360. "amour je tai tue" trad. "amore ti ho ucciso" (corsivo dell'autore).

35. Ib., p. 411, "chance" trad. "possibilita" (corsivo dell'autore). 
passato il tempo in cui nello slip

ti guardavi contenta, accontentata

d'un umore qualsiasi

follie-bergères dietro ogni

mobile, spostabile definitivamente

ogni mattina ${ }^{36}$

Come se sapessi cosa vuol dire l'opposto

cose lontanissime nella piccola patria

outside la foresta, e dai tropicali mucchi

nel beige del tricolore

morgana dalle ali incorrotte... ${ }^{37}$

Perché morendo ci fai venir a festa? Semmai

era l'altro lato che andava premiato

e tu non rifiutasti quel cibo acerbo

vinaigre di festa e botte sulle spalle... ${ }^{38}$

Non v'è sole che non sia

lumière, (e il francese ̀̀

un par terre) quando cangiando

viste, cangiasti forme, anche...

Per chiarire il concetto

mi distinsi poi, non prima:

mentre voi vi facevate la

toletta, toilette nel dormitorio

più colto dei vostri sogni.

da quando tu

mi licenziasti m'abbevvi

36. Amelia Rosselli, Documento, in Le poesie, op. cit., p. 574. slip... follie-bergères" trad. "slip/mutanda... folliebergères' (corsivi dell'autore).

37. Ib., p. 588, "outside... beige" trad. "fuori... beige" (corsivo dell'autore).

38. Ib., p. 614, "vinaigre" trad. "aceto" (corsivo dell'autore). 
di tè, quale è il colloquio

ripido di cui mi ricordavate

sostenendone la tesi, sipping,

or drilling, sollevando insomma

il labbro la tazza al mento...

vostro odore di santità politica

nel campo, dove riposo ingiunta

dal massacro a massacrarmi

come voi, nei desideri intensi

curvati nel blé, the grass

che vi fa invidia perché... ${ }^{39}$

Questi inserti stranieri sono le punte visibili di un vissuto linguistico rimosso, ma che tuttavia preme e ogni tanto fa sentire la sua voce, inserendosi in un quadro di devianze tali per cui anche l'italiano di Amelia sembra una lingua straniera, estranea, strana, una lingua dell'altro mondo. Ed è incredibile come questa lingua inventata, quest'altra lingua, possieda un'eccezionale forza di pensiero e una grande potenza "comunicativa" Tra apertura e chiusura, questa "scrittura del rischio" testimonia il suo essere nel mondo e nel contempo l'impossibilità di essere nel mondo. Ė una scommessa, un sfida all'incomunicabilità, un tentativo di entrare in contatto con gli altri senza tradire se stessa.

Ha scritto Maria Clelia Cardona: "Ciò che subito colpiva in lei era la perfetta coincidenza del suo essere con il suo linguaggio poetico. La forza di quel linguaggio consisteva (consiste) nell'escludere turti gli altri. [...] Per Amelia la poesia non era un esercizio da tavolino, o un modo per abbellire o riempire la vita; non era un gioco, né un linguaggio cifrato da praticare nel tempo libero, come tanto spesso accade:

39. Amelia Rosselli, Imprompeu, in Le poesie, op. cit., pp. 643-646-647-648, "lumière... par terre... toilette... sipping, or drilling... blé... che grass" trad. "luce... parterre... toilette... sorseggiando, o trapanando... grano... l'erba" (corsivi dell'autore). 
era il suo linguaggio e la sua natura" Se questo è vero, allora è vero che per Amelia la creazione della propria lingua poetica è la creazione della sua stessa identità. Il suo linguaggio dice "io" a suo modo. Da qui l'impressione che si ha, leggendo la sua poesia, di imbattersi in un discorso imperioso, che lotta tra ordine e disordine, che si svolge da se stesso per una sua storia interna.

In Sleep Amelia aveva scritto: "la vita scritta su carta, là scorre il mio seme folle alla morte" e in Documento: "Lo scritto che in me è folle risponde a tutto questo dolore con parole sempre spero sempre vere" Per Amelia, per questa apolide, per questa senzapatria, esule dovunque, "schiodare il verso", ritagliarsi la propria lingua, è stata l'unica forma di appartenenza in cui si è riconosciuta, un tentativo di aggrapparsi al mondo e di bucare l'involucro della propria solitudine. Essere poeta era il suo modo di stare nel mondo. Ė stato più volte affermato da chi l'ha conosciuta che Amelia pensava in poesia, che anche nel quotidiano creava immagini e inventava parole. In lei, lingua della vita e lingua della poesia coincidevano.

C'è un aspetto altamente drammatico in questa identità di scrittura e vita chiusa tra due tragedie, quella iniziale dell'assassinio del padre e quella finale del suo suicidio, perciò questa poesia fa sembrare inadatte e vuote le parole della letteratura riducendole spesso all'impotenza.

Più che una scrittura, la sua sembra essere una voce che esce fuori dalla pagina e ci tocca, e a noi non resta che cercare di ascoltare quella voce e sopportare quel contatto senza restarne ustionati. La sua poesia è un corpo che parla.

RESUMO: Em Amelia Rosselli, a busca de uma lingua "própria" se deu com rigor e tensāo dramática, mas também com jogo e leveza, coincidindo com a busca de sua própria identidade. Entre aberturas e fechamentos, a lingua de Amelia Rosselli se constitui em corpo sólido e revela sua origem trilingüe. PALAVRAS-CHAVE: Amelia Rosselli (Paris 1930-Roma 1996); poesia; identidade; lingua, trilingüismo. 